Received: 13 April 2017

Accepted: 22 November 2017

Published online: 04 December 2017

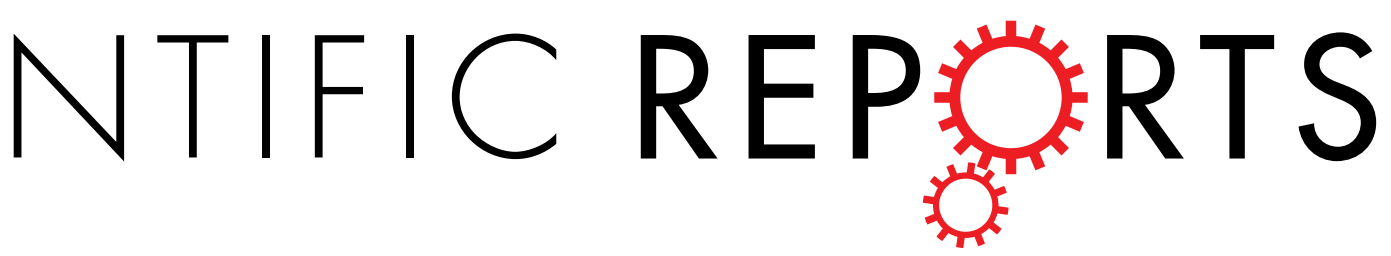

\title{
Quantitative Analysis of
} Inflammation in Orbital Fat of Thyroid-associated

\section{Ophthalmopathy Using MRI Signal Intensity}

\author{
Tomoaki Higashiyama, Maki Iwasa \& Masahito Ohji
}

We quantitatively analyzed inflammation in orbital fat from cases of thyroid-associated ophthalmopathy (TAO) using short-tau inversion recovery (STIR) sequences from magnetic resonance imaging. The signal intensity ratios (SIRs) of orbital fat as well as the superior rectus (SR), inferior rectus (IR), lateral rectus (LR), medial rectus (MR), and superior oblique (SO) muscles on STIR images were measured in 70 eyes from 70 treatment-naive TAO patients (active TAO group, 19 patients; inactive TAO group, 51 patients) and 20 eyes from 20 controls. The mean SIR in the active TAO group was significantly higher than that in the inactive TAO group and controls $(P<0.001)$. The SIR of orbital fat in all TAO patients was significantly $(P<0.001)$ positively correlated with that of the extraocular muscles: SR $(r=0.64)$, IR $(r=0.55), \operatorname{LR}(r=0.58), \mathrm{MR}(r=0.71)$, and SO $(r=0.65)$. The SIR of orbital fat had a significant positive correlation with the CAS $(r=0.53, P<0.001)$. The measurements of SIRs in orbital fat may be useful in evaluating the activity in tissues of TAO patients.

Thyroid-associated ophthalmopathy (TAO) is the most prevalent extra-thyroidal manifestation of thyroid disease $^{1}$. TAO is an immune-mediated inflammatory disease in which the levels of anti-thyroid antibodies are elevated. The disease causes increased orbital fat and enlarged extraocular muscles in the orbit ${ }^{1,2}$. Some clinical signs and symptoms of TAO arise from enlarged orbital tissues ${ }^{2-7}$. TAO has previously been classified as either the predominantly increased orbital fat type or predominantly enlarged extraocular muscle type ${ }^{5,8}$. Younger patients tend to have increased orbital fat, whereas older patients are more likely to have enlarged extraocular muscles ${ }^{2}$.

We previously reported the quantitative evaluation of inflammation in the extraocular muscles of active TAO patients using short-tau inversion recovery (STIR) sequences of magnetic resonance imaging (MRI) ${ }^{9}$. Inflammation can be quantitatively evaluated in STIR images because the high signal intensity indicates edema caused by inflammation in extraocular muscles ${ }^{10-16}$. Our previous report demonstrated that the pretreatment signal intensity in the extraocular muscles of TAO patients was significantly higher than that in controls by MRI ${ }^{9}$. The signal intensity after methylprednisolone pulse therapy was significantly lower than the pretreatment intensity. In addition, the signal intensity in patients with TAO was significantly correlated with the clinical activity score (CAS). Another study also obtained similar findings ${ }^{15}$.

However, inflammation in the orbital fat of TAO patients has not been studied quantitatively, which has left some question unanswered, such as the comparison of the signal intensities in orbital fat among active TAO patients, inactive TAO patients, and controls as well as the correlation of the inflammation in orbital fat with the inflammation in the extraocular muscles, CAS, and patient's age.

Thus, we investigated the signal intensity of orbital fat in TAO patients and controls using the MRI STIR sequences.

\section{Results}

Comparison of the orbital fat signal intensity ratios (SIRs) among the active TAO group, inactive TAO group, and controls. The active (CAS $\geq 3 / 7)$ TAO group included 19 patients, and the inactive 


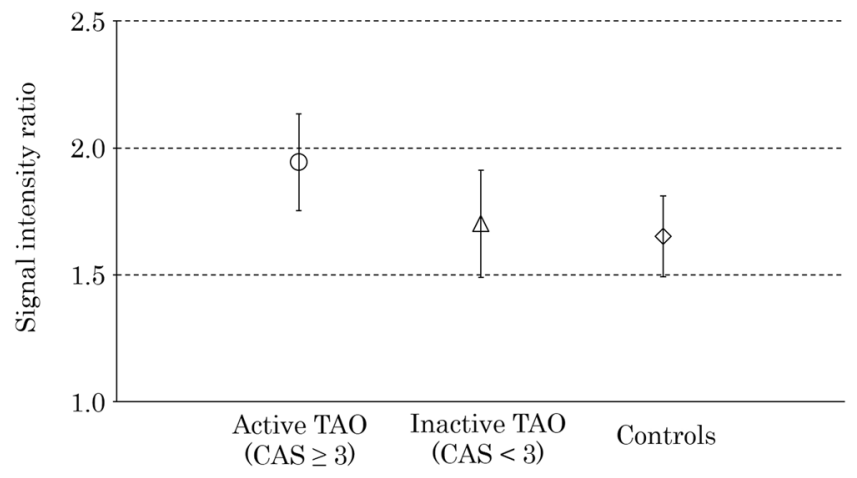

Figure 1. Comparison of the orbital fat signal intensity ratio (SIR) among the active thyroid-associated ophthalmopathy (TAO) group, inactive TAO group, and controls. The plots and bars of the active TAO group (circle), inactive TAO group (triangle) and controls (diamond) indicate the mean and standard deviation. The mean SIR in the active TAO group was significantly higher than that in the inactive TAO group and controls $(P<0.001)$. The mean SIR in the inactive TAO group was not significantly higher than that in the controls $(P=0.62)$.

\begin{tabular}{|l|c|c|c|c|c|c|}
\hline \multirow{2}{*}{} & \multirow{2}{*}{} & & & \multicolumn{3}{|c|}{ P value } \\
\cline { 5 - 7 } & Active TAO & Inactive TAO & Controls & $\begin{array}{c}\text { Active TAO vs. } \\
\text { Inactive TAO }\end{array}$ & $\begin{array}{c}\text { Active TAO } \\
\text { vs. Controls }\end{array}$ & $\begin{array}{c}\text { Inactive TAO } \\
\text { vs. Controls }\end{array}$ \\
\hline FAT & $1.94 \pm 0.19$ & $1.70 \pm 0.21$ & $1.65 \pm 0.16$ & $<0.001$ & $<0.001$ & 0.62 \\
\hline SR & $2.56 \pm 0.70$ & $1.78 \pm 0.62$ & $1.08 \pm 0.25$ & $<0.001$ & $<0.001$ & $<0.001$ \\
\hline IR & $2.76 \pm 0.72$ & $2.03 \pm 0.58$ & $1.32 \pm 0.28$ & $<0.001$ & $<0.001$ & $<0.001$ \\
\hline LR & $2.14 \pm 0.45$ & $1.75 \pm 0.32$ & $1.34 \pm 0.18$ & $<0.001$ & $<0.001$ & $<0.001$ \\
\hline MR & $2.61 \pm 0.53$ & $1.91 \pm 0.36$ & $1.47 \pm 0.24$ & $<0.001$ & $<0.001$ & $<0.001$ \\
\hline SO & $2.14 \pm 0.47$ & $1.58 \pm 0.30$ & $1.26 \pm 0.22$ & $<0.001$ & $<0.001$ & $<0.001$ \\
\hline
\end{tabular}

Table 1. Comparison among the signal intensity ratios of orbital fat in the active thyroid-associated ophthalmopathy (TAO) group, inactive TAO group, and controls. TAO = thyroid-associated ophthalmopathy; $\mathrm{FAT}=$ orbital fat, $\mathrm{SR}=$ superior rectus; $\mathrm{IR}=$ inferior rectus; $\mathrm{LR}=$ lateral rectus; $\mathrm{MR}=$ medial rectus; $\mathrm{SO}=$ superior oblique.

TAO group included 51 patients. The mean CASs were $3.74 \pm 0.73$ (range, $3-5$ ) in the inactive TAO group and $1.00 \pm 0.85$ (range, $0-2$ ) in the inactive TAO group.

The mean orbital fat SIRs were $1.94 \pm 0.19$ in the active TAO group, $1.70 \pm 0.21$ in the inactive TAO group, and $1.65 \pm 0.16$ in the controls. The mean SIR in the active TAO group was significantly higher than that in the inactive TAO group and controls $(P<0.001)$ (Fig. 1, Table 1). The mean SIR in the inactive TAO patients was not significantly higher than that in the controls $(P=0.62)$. Table 1 also shows the comparison of the extraocular muscle SIRs among the groups.

Correlations between the orbital fat SIRs and the extraocular muscle SIRs. The mean SIRs in all TAO patients were $1.99 \pm 0.73,2.23 \pm 0.69,1.86 \pm 0.39,2.10 \pm 0.51$ and $1.73 \pm 0.43$ in the superior rectus (SR), inferior rectus (IR), lateral rectus (LR), medial rectus (MR), and superior oblique (SO) muscles, respectively. The orbital fat SIRs showed significant $(P<0.001)$ positive correlations with those of the extraocular muscles: SR $(r=0.64)$, IR $(r=0.55)$, LR $(r=0.58)$, MR $(r=0.71)$ and SO $(r=0.65)$ (Fig. 2, Table 2$)$.

Correlation between orbital fat SIRs and the CAS. The correlation between the orbital fat SIR and the CAS was analyzed. The mean value in all TAO patients was $1.74 \pm 1.47$. The orbital fat SIR showed a significant positive correlation with the CAS $(r=0.53, P<0.001)$ (Fig. 3$)$.

Correlations between orbital fat SIRs and age. A significant correlation was not observed between the orbital fat SIR and age $(r=0.19, P=0.11)$ (Fig. 4A). Very weak significant positive correlations were observed between the SIRs of some of the extraocular muscles and age: IR $(r=0.41, P<0.001)$, LR $(r=0.24, P=0.047)$, $\operatorname{MR}(r=0.36, P=0.002)$ (Fig. $4 \mathrm{~B})$.

The mean ages were $41.5 \pm 8.6$ years (range $14-50$ years) in the young group ( 36 patients) and $62.6 \pm 6.2$ years (range 51-78 years) in the old group (34 patients). Very weak significant positive correlations were observed between the SIRs of some of the extraocular muscles and age in the young group: SR $(r=0.46, P=0.005)$, IR $(r=0.38, P=0.23), \operatorname{LR}(r=0.34, P=0.04)$ and $\mathrm{MR}(r=0.34, P=0.04)$. However, a significant correlation was not observed between the extraocular muscles SIRs and age in the old group (Table 3). Significant correlations were not observed between the orbital fat SIR and age in either group. 

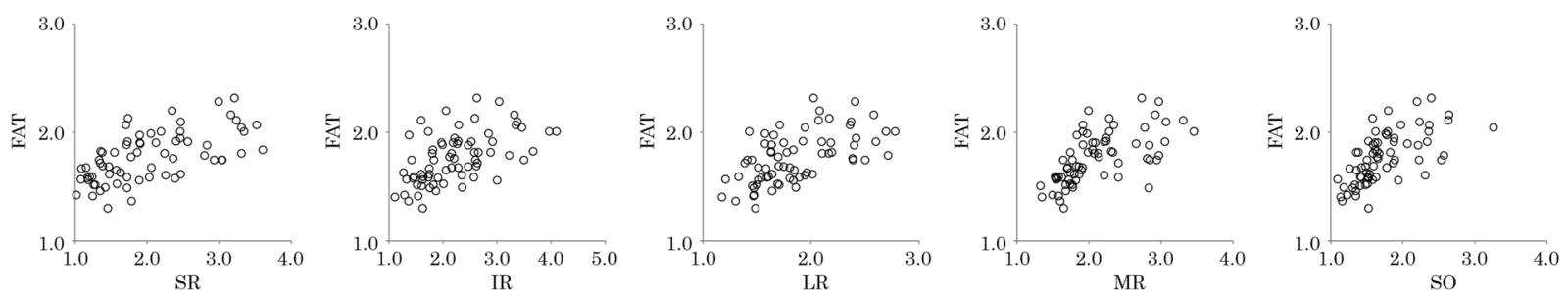

Figure 2. Correlation between the signal intensity ratio (SIR) of the orbital fat and those of extraocular muscles. The orbital fat SIR showed significant $(P<0.001)$ positive correlations with those of extraocular muscles: SR $(r=0.64)$, IR $(r=0.55), \mathrm{LR}(r=0.58), \mathrm{MR}(r=0.71)$, and SO $(r=0.65)$. FAT $=$ orbital fat; $\mathrm{SR}=$ superior rectus; $\mathrm{IR}=$ inferior rectus; $\mathrm{LR}=$ lateral rectus; $\mathrm{MR}=$ medial rectus; $\mathrm{SO}=$ superior oblique.

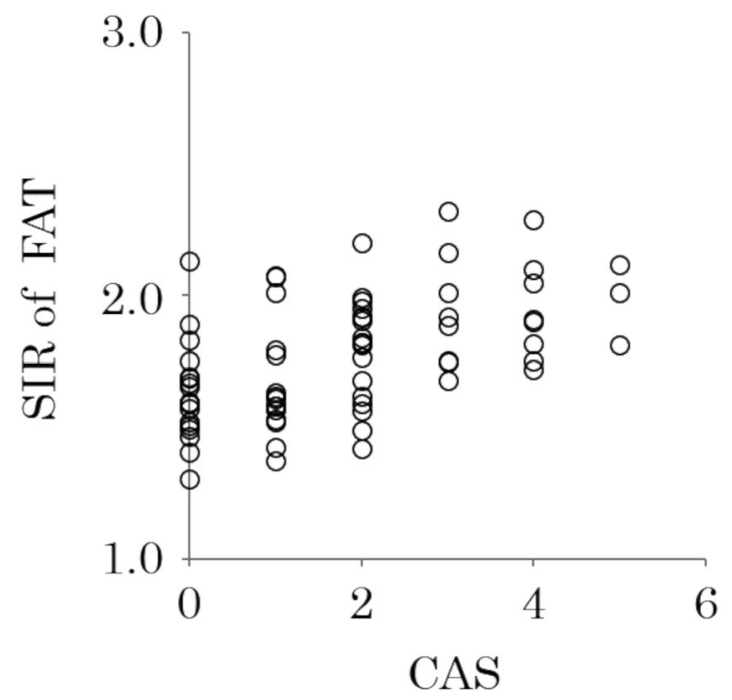

Figure 3. Correlation between the orbital fat signal intensity ratio (SIR) and clinical activity scores. The orbital fat SIR showed a significant positive correlation with the Clinical Activity Score (CAS) $(r=0.53, P<0.001)$. SIR of FAT $=$ signal intensity ratio of orbital fat.

\begin{tabular}{|l|c|c|}
\hline & r & Pvalue \\
\hline SR & 0.64 & $<0.001$ \\
\hline IR & 0.55 & $<0.001$ \\
\hline LR & 0.58 & $<0.001$ \\
\hline MR & 0.71 & $<0.001$ \\
\hline SO & 0.65 & $<0.001$ \\
\hline
\end{tabular}

Table 2. Correlations between orbital fat and extraocular muscle signal intensity ratios (SIRs). FAT $=$ orbital fat, $\mathrm{SR}=$ superior rectus; $\mathrm{IR}=$ inferior rectus; $\mathrm{LR}=$ lateral rectus; $\mathrm{MR}=$ medial rectus; $\mathrm{SO}=$ superior oblique.

\section{Discussion}

The mean SIR in the active TAO group was significantly higher than that in the inactive TAO group and controls in this study. Chen et al. reported a comparison of macrophage infiltration into orbital fat between TAO patients and controls ${ }^{17}$. These authors showed that macrophage infiltration into orbital fat was greater in TAO patients than in controls. Histological examination to quantitatively evaluate inflammation in the orbital fat in a clinical setting, however, is almost impossible for many TAO patients. Hence, the noninvasive use of MRI STIR sequences to quantitatively evaluate the signal intensity of orbital fat inflammation may be useful in TAO patients. The current study revealed that orbital fat inflammation may develop due to increased disease activity in TAO patients.

A significant positive correlation was observed between the SIR of orbital fat and that of the extraocular muscles in this study. Other studies previously reported that TAO patients could be classified as having predominantly increased orbital fat or predominantly enlarged extraocular muscles ${ }^{5,8}$. Hiromatsu et al. investigated the cytokine profiles of orbital fat and extraocular muscle tissues in TAO patients ${ }^{8}$. These authors reported that the cytokine profiles were different between these two tissue sources. Kuriyan et al. investigated the effect of cyclooxygenase inhibitors on adipogenesis and proliferation of orbital fibroblasts in the two TAO subtypes (the predominantly fat 
(A)

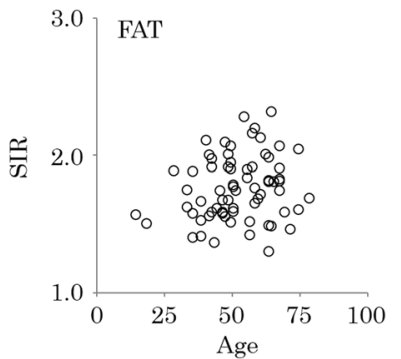

(B)

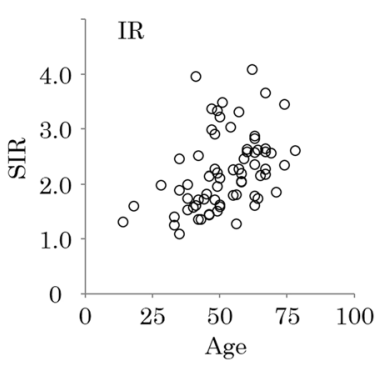

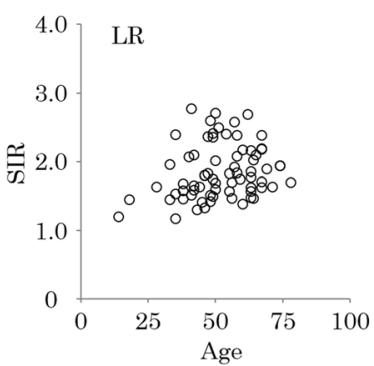

Figure 4. Correlation of age with the signal intensity ratio (SIR) of orbital fat and extraocular muscles. The orbital fat SIR did not show a significant correlation with age $(r=0.19, P=0.11)(\mathbf{A})$. The SIRs of some extraocular muscles showed significant positive correlations with age: IR $(r=0.42, P<0.001), \operatorname{LR}(r=0.24$, $P=0.047)$ and $\mathrm{MR}(r=0.36, P=0.002)(\mathrm{B}) . \mathrm{FAT}=$ orbital fat; $\mathrm{IR}=$ inferior rectus; $\mathrm{LR}=$ lateral rectus; $\mathrm{MR}=$ medial rectus.

\begin{tabular}{|l|c|c|c|c|}
\hline \multirow{2}{*}{} & \multicolumn{2}{|c|}{ Young group } & \multicolumn{2}{c|}{ Old group } \\
\cline { 2 - 5 } & r & P value & r & P value \\
\hline FAT & 0.28 & 0.10 & -0.15 & 0.39 \\
\hline SR & 0.46 & 0.05 & -0.14 & 0.42 \\
\hline IR & 0.38 & 0.02 & 0.09 & 0.60 \\
\hline LR & 0.34 & 0.04 & -0.17 & 0.33 \\
\hline MR & 0.34 & 0.04 & -0.16 & 0.37 \\
\hline SO & 0.32 & 0.06 & 0.11 & 0.55 \\
\hline
\end{tabular}

Table 3. Correlations between orbital fat and extraocular muscle signal intensity ratios (SIRs) and age. $\mathrm{FAT}=$ orbital fat, $\mathrm{SR}=$ superior rectus; $\mathrm{IR}=$ inferior rectus; $\mathrm{LR}=$ lateral rectus; $\mathrm{MR}=$ medial rectus; $\mathrm{SO}=$ superior oblique.

compartment enlargement type and predominantly extraocular muscle enlargement type $)^{5}$. Orbital fibroblasts were isolated and established from TAO patients undergoing orbital decompression. The authors reported that the effect of cyclooxygenase inhibitors was different for the two subtypes because the cyclooxygenase inhibitors significantly decreased proliferation and adipogenesis in the orbital fibroblasts of patients with the predominantly extraocular muscle enlargement type but not the predominantly fat compartment enlargement type. In contrast, our study showed that the orbital fat and extraocular muscle SIRs were significantly correlated. We showed that the orbital fat inflammation might develop in conjunction with that in extraocular muscles, although the pathological pathways in these tissues might be different.

The orbital fat SIR showed a significant positive correlation with the CAS in this study. We previously reported a significant positive correlation between the extraocular muscle SIRs and CAS in TAO patients ${ }^{9}$. Another study also reported that a significant positive correlation was found between the extracocular muscle SIRs and the Mourits score, which is the basis for the CAS in TAO patients ${ }^{14}$. Our current study revealed that the orbital fat SIR was also correlated with the CAS.

A significant correlation was not found between the orbital fat SIR and age, whereas a very weak significant positive correlation was observed between some of the extraocular muscle SIRs and age. In other review articles, younger patients were more likely to exhibit increased orbital fat without extraocular muscle enlargement, whereas older patients were more prone to exhibit severe extraocular muscle enlargement ${ }^{2,18,19}$. Murakami et al. investigated the relationship between age and extraocular muscle enlargement by CT in TAO patients ${ }^{20}$. The frequency of extraocular muscle enlargement was correlated with age in older patients, which agreed with our results. To the best of our knowledge, the relationship between age and orbital fat inflammation has not been previously reported. The current study showed that the SIR of orbital fat did was not significantly correlated with age. Inflammation in the fat might not be strongly affected by age, unlike that in the extraocular muscles.

The present study has several limitations. First, the sample size was small, with only 70 patients enrolled. Second, the mean CAS was low. It is known that the clinical signs of TAO differ among races, and the CAS tends to be low in Japanese patients with $\mathrm{TAO}^{21}$, which could be the reason for the low CAS observed in this study.

In conclusion, measuring the orbital fat SIR may be useful for evaluating disease activity in TAO patients.

\section{Methods}

Subjects. The institutional review board of Shiga University of Medical Science approved this retrospective study. The study adhered to the tenets of the Declaration of Helsinki. Each patient provided written informed consent.

In total, 70 eyes from 70 patients with TAO ( 52 female, 18 male; mean \pm SD age $51.8 \pm 13.0$ years, range $14-78$ years) and 20 eyes from 20 volunteer controls ( 4 female, 16 male; mean age $36.8 \pm 11.3$ years, range $20-54$ years) 

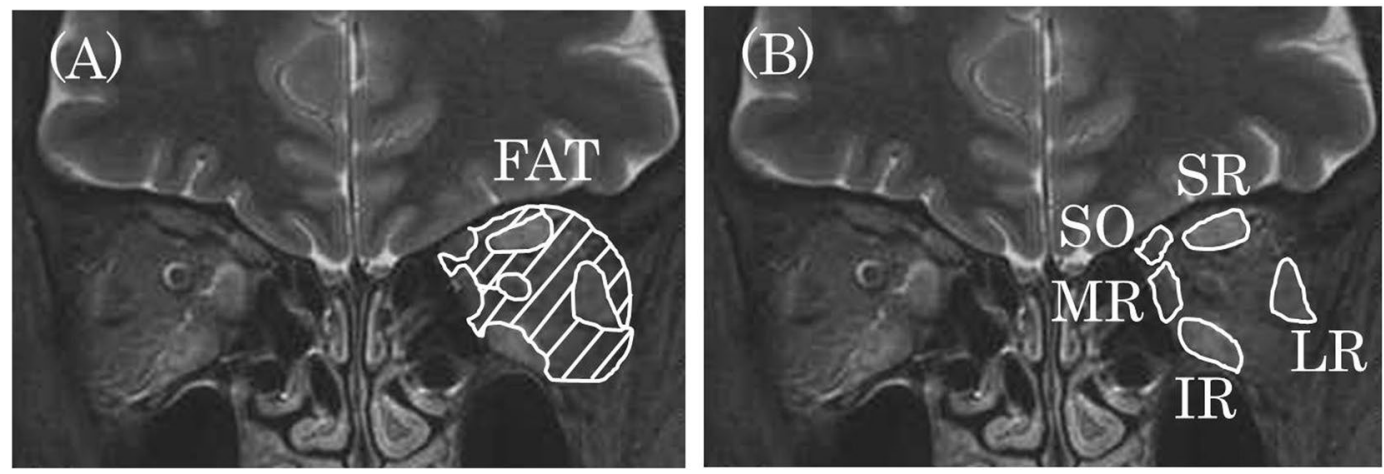

Figure 5. Measurement of signal intensities of orbital fat and extraocular muscles. The signal intensities of orbital fat (A) and each muscle (B) were measured, as shown, in the left orbit and brain. FAT = orbital fat, $\mathrm{SR}=$ superior rectus; $\mathrm{IR}=$ inferior rectus; $\mathrm{LR}=$ lateral rectus; $\mathrm{MR}=$ medial rectus; $\mathrm{SO}=$ superior oblique.

were included in this study at the Department of Ophthalmology, Shiga University of Medical Science Hospital, from April 2008 to December 2016. The eye in which the mean orbital fat SIR was higher was selected as representative of the patient, and the right eye was selected as representative of the control. Only treatment-naive TAO patients who had a high level of serum thyroid autoantibodies were included. The normal serum thyroid autoantibody ranges are as follows: thyrotropin receptor antibody determined by the first and second generation assays (first normal: 0-15\%, second normal: 0-0.9 IU/L), thyroid stimulating antibody (normal 0-120\%), thyroid peroxidase antibody (normal 0-15.9 IU/mL), and thyroglobulin antibody (normal 0-27.9 IU/mL). If patients had an orbital disease or an inflammatory disease in the orbit, they were excluded.

Signal intensity measurements. All patients were subjected to orbital MRI with a standard head coil. The MRI was performed on a 3.0-tesla MRI unit (Achieva 3.0 T Quasar Dual; Royal Philips Electronics, Amsterdam, The Netherlands).

The signal intensities of the orbital fat and the SR, IR, LR, MR, and SO muscles were measured in the STIR and T2-weighted images (Fig. 5). The regions of interest (ROIs) were drawn around the tissues on coronal T2-weighted images. The drawn ROIs were superimposed on the STIR images at the same level, and the signal intensities were measured.

To standardize the signal intensity in each tissue, the signal intensity of brain white matter (WM) on STIR images was measured as the "standard" tissue, which was similar to the protocol described in our previous studies $^{9,16}$. The SIRs between the orbital fat or extraocular muscles and the WM of the same slice was calculated. The highest value among the mean SIRs of three consecutive slices in each tissue was considered representative of the SIR for that tissue.

Assessment of CAS. The CAS (maximum score 7 points) was measured in all TAO patients. The CAS consists of seven findings: spontaneous retrobulbar pain; pain on attempted up or down gaze; redness of the conjunctiva; redness of the eyelids; inflammation of the caruncle and/or plica; swelling of the eyelids; and conjunctival edema $^{22,23}$. Patients with CAS $\geq 3 / 7$ were considered as having active $\mathrm{TAO}^{22}$. TAO patients were divided into two groups (the active CAS group and inactive CAS group) in the comparison analysis.

Statistical analysis. All statistical analyses were performed using SPSS Statistics 22 software (IBM, Armonk, NY, USA). The normality of the numerical variables was evaluated using the Shapiro-Wilk test. The Tukey HSD test was used to compare the SIRs among the active TAO group, inactive TAO group, and controls. Pearson's product-moment correlation coefficient or Spearman's rank correlation coefficient were used to analyze the correlations between the orbital fat SIRs and those of extraocular muscles and the correlations between the SIRs and $\mathrm{CAS}$ and age in all TAO patients. The values are expressed as the mean $\pm \mathrm{SD}$. A $P$ value of less than 0.05 indicated statistical significance.

\section{References}

1. Barrio-Barrio, J., Sabater, A. L., Bonet-Farriol, E., Velázquez-Villoria, Á. \& Galofré, J. C. Graves' Ophthalmopathy: VISA versus EUGOGO Classification, Assessment, and Management. J Ophthalmol 2015, 249125 (2015).

2. Bahn, R. S. Graves'ophthalmopathy. N Engl J Med 362, 726-738 (2010).

3. Prummel, M. F. et al. Multi-center study on the characteristics and treatment strategies of patients with Graves' orbitopathy: the first European Group on Graves' Orbitopathy experience. Eur J Endocrinol 148, 491-495 (2003).

4. Kuriyan, A. E., Phipps, R. P. \& Feldon, S. E. The eye and thyroid disease. Curr Opin Ophthalmol 19, 499-506 (2008).

5. Kuriyan, A. E., Woeller, C. F., O’Loughlin, C. W., Phipps, R. P. \& Feldon, S. E. Orbital fibroblasts from thyroid eye disease patients differ in proliferative and adipogenic responses depending on disease subtype. Invest Ophthalmol Vis Sci 54,7370-7377 (2013).

6. Nishida, Y. et al. Significance of orbital fatty tissue for exophthalmos in thyroid-associated ophthalmopathy. Graefes Arch Clin Exp Ophthalmol 240, 515-520 (2002).

7. Higashiyama, T., Nishida, Y. \& Ohji, M. Changes of orbital tissue volumes and proptosis in patients with thyroid extraocular muscle swelling after methylprednisolone pulse therapy. Jpn J Ophthalmol 59, 430-435 (2015).

8. Hiromatsu, Y. et al. Cytokine profiles in eye muscle tissue and orbital fat tissue from patients with thyroid-associated ophthalmopathy. J Clin Endocrinol Metab 85, 1194-1199 (2000). 
9. Higashiyama, T. et al. Use of MRI signal intensity of extraocular muscles to evaluate methylprednisolone pulse therapy in thyroidassociated ophthalmopathy. Jpn J Ophthalmol 59, 124-130 (2015).

10. Hiromatsu, Y. et al. Role of magnetic resonance imaging in thyroid-associated ophthalmopathy: its predictive value for therapeutic outcome of immunosuppressive therapy. Thyroid 2, 299-305 (1992).

11. Hoh, H. B. et al. The STIR sequence MRI in the assessment of extraocular muscles in thyroid eye disease. Eye (Lond) 8, 506-510 (1994).

12. Laitt, R. D. et al. The value of the short tau inversion recovery sequence in magnetic resonance imaging of thyroid eye disease. $\mathrm{BrJ}$ Radiol 67, 244-247 (1994).

13. Bailey, C. C. et al. Magnetic resonance imaging in thyroid eye disease. Eye (Lond) 10, 617-619 (1996).

14. Mayer, E. et al. Serial STIR magnetic resonance imaging correlates with clinical score of activity in thyroid eye disease. Eye (Lond) 15, 313-318 (2001)

15. Tortora, F. et al. Diagnostic accuracy of short-time inversion recovery sequence in Graves' Ophthalmopathy before and after prednisone treatment. Neuroradiology 56, 353-361 (2014).

16. Higashiyama, T., Nishida, Y. \& Ohji, M. Relationship between MRI Signal Intensity and Volume of Extraocular Muscles in ThyroidAssociated Ophthalmopathy with Methylprednisolone Pulse Therapy. Clin Ophthalmol 10, 721-729 (2016).

17. Chen, M. H., Chen, M. H., Liao, S. L., Chang, T. C. \& Chuang, L. M. Role of macrophage infiltration in the orbital fat of patients with Graves' ophthalmopathy. Clin Endocrinol (Oxf) 69, 332-337 (2008).

18. Bahn, R. S. Clinical review 157: Pathophysiology of Graves' ophthalmopathy: the cycle of disease. J Clin Endocrinol Metab 88, 1939-1946 (2003).

19. Hiromatsu, Y., Eguchi, H., Tani, J., Kasaoka, M. \& Teshima, Y. Graves'ophthalmopathy: epidemiology and natural history. Intern Med 53, 353-360 (2014).

20. Murakami, Y., Kanamoto, T., Tuboi, T., Maeda, T. \& Inoue, Y. Evaluation of extraocular muscle enlargement in dysthyroid ophthalmopathy. Jpn J Ophthalmol 45, 622-627 (2001).

21. Tachibana, S. et al. Orbital magnetic resonance imaging combined with clinical activity score can improve the sensitivity of detection of disease activity and prediction of response to immunosuppressive therapy for Graves' ophthalmopathy. Endocr J. 57, 853-861 (2010).

22. Bartalena, L. et al. Consensus statement of the European Group on Graves' Orbitopathy (EUGOGO) on management of GO. Eur J Endocrinol 158, 273-285 (2008).

23. Bartalena, L. et al. The 2016 European Thyroid Association/European Group on Graves' Orbitopathy Guidelines for the Management of Graves' Orbitopathy. Eur Thyroid J. 5, 9-26 (2016).

\section{Acknowledgements}

We thank Dr. Yasuhiro Nishida for various types of support during this study.

\section{Author Contributions}

Conceived and designed the experiments, T.H., M.I. and M.O.; performed the experiments, T.H.; analyzed the data, T.H., M.I. and M.O.; contributed the materials, T.H., M.I. and M.O.; and wrote the paper, T.H., M.I. and M.O.

\section{Additional Information}

Competing Interests: The authors declare that they have no competing interests.

Publisher's note: Springer Nature remains neutral with regard to jurisdictional claims in published maps and institutional affiliations.

Open Access This article is licensed under a Creative Commons Attribution 4.0 International License, which permits use, sharing, adaptation, distribution and reproduction in any medium or format, as long as you give appropriate credit to the original author(s) and the source, provide a link to the Creative Commons license, and indicate if changes were made. The images or other third party material in this article are included in the article's Creative Commons license, unless indicated otherwise in a credit line to the material. If material is not included in the article's Creative Commons license and your intended use is not permitted by statutory regulation or exceeds the permitted use, you will need to obtain permission directly from the copyright holder. To view a copy of this license, visit http://creativecommons.org/licenses/by/4.0/.

(C) The Author(s) 2017 